Communications in Physics, Vol.21, No. 3 (2011), pp. 265-271

\title{
THE SURROUNDED ATOM THEORY OF ORDER-DISORDER PHASE TRANSITION IN BINARY ALLOYS
}

\author{
DO CHIEU HA \\ Saigon University \\ NGUYEN NHAT KHANH \\ Department of Physics, University of Natural Sciences, \\ Ho Chi Minh City National University
}

\begin{abstract}
In this paper, the surrounded atom model is developed to study the order-disorder phase transition in binary alloys. We calculate the configurational free energy of the alloys, derive the equation of equilibrium and determine the critical temperature of the phase transition.
\end{abstract}

\section{INTRODUCTION}

Order-disorder transitions have been an active field of research over decades. The first model for order- disorder transition in binary alloys was the Bragg-Williams model [1-3], that suggested the existence of a long-range order parameter. In 1938 Kirkwood presented a more general method by which the configurational free energy can be expanded as a series in the long-range order parameter [3]. However, both Bragg-Williams and Kirkwood models which incorporated only pair interactions predicted a higher transition temperature and incorrect specific heat capacity above transition temperature [1-3]. The numerical approaches have been proposed to take into account the other interactions and fluctuations: ab-initio calculations, Monte Carlo simulations [5]. The studies of phase transitions based on numerical methods have proved difficulties, especially when magnetic orders and other interactions were taken into account. Therefore it is desired to develop some simple analytical models, which can go beyond the pair interaction approximation. In 1967 Bonnier et al proposed the surrounded atom model as a gereralization of the quasichemical method for calculating thermodynamic quantities of binary alloys [6]. Thus it is meaningful to employ the surrounded atom model to study order-disorder phase transitions in binary alloys.

In the present work we use the approach suggested by Bonnier et al for calculating configurational free energy of binary alloys and obtaining the critical temperature and the heat capacity at the critical temperature.

The paper is organized as follows. In Section II the formalism is introduced. The configurational energy is calculated, then the critical temperature and the heat capacity are determined. The discussions are presented in the final section, where we compared our results with ones obtained by other known methods. 


\section{CONFIGURATIONAL FREE ENERGY AND ORDER PARAMETERS}

Consider a binary order-disorder alloy with composition $A_{m} B_{n}$ having two kinds of atoms $\mathrm{A}$ and $\mathrm{B}$ on a lattice. Let $Z$ be the coordination number of the lattice, $N$ - the number of lattice sites and $N_{A}$ and $N_{B}$ - the number of $\mathrm{A}$ and $\mathrm{B}$ atoms, respectively. Each A-site has $Z_{A A}$ nearest neighbours A-atoms and $Z_{A B}$ nearest neighbours B-atoms; $Z_{B B}$ and $Z_{B A}$ are similarly defined. We define the quantities $N_{A A}, N_{B B}$ and $N_{B A}$ to be the number of nearest neighbours pairs of the AA, BB and $\mathrm{AB}$ type, respectively, and we assume each pair has the interaction energy $V_{A A}, V_{A B}$ and $V_{B B}$, respectively, for $\mathrm{AA}, \mathrm{AB}$ and $\mathrm{BB}$ bond. At absolute zero temperature the alloy is completely ordered. Suppose that the lattice of alloy on perfectly ordered state is devided into two sublattices of labels $a$ and $b$. Then the number of A atoms on $a$ sites $N_{A a}$ is equal to $N_{A}$ and the number of $\mathrm{B}$ atoms on $b$ sites is equal to $N_{B}$. At a given temperature, the free energy is a minimum and a balance between the energy and the entropy is established so the crystal is partially ordered and $N_{A a}<N_{A} ; N_{B b}<N_{B}$. The configuration of the system is defined by numbering the lattice sites and specifying the type of atom on each site. For a given set of occupation number $\left\{N_{A a}, N_{B b}\right\}$ there are a great many distinguishable configurational states. The long range order is measured by the parameter [1-3]:

$$
\eta=\frac{\mathrm{f}_{\mathrm{Aa}}-C_{A}}{1-C_{A}}
$$

where $f_{A a}$ means the fraction of $\mathrm{A}$ atoms in the $a$ sublattices and $C_{\alpha}(\alpha=\mathrm{A}, \mathrm{B})$ is the fraction of $\alpha$ atoms in the crystal. In this way $\eta=1$ for the ordered phase and $\eta=0$ for the disordered phase.

Concerning the short-range correlations one introduces the short-range order parameter as [3].

$$
\sigma=\frac{\mathrm{q}-\mathrm{q}_{0}}{\mathrm{q}_{\mathrm{m}}-\mathrm{q}_{0}}
$$

where $\mathrm{q}$ is the fraction of unlike bonds, $\mathrm{q}_{\mathrm{m}}\left(\mathrm{q}_{0}\right)$ is its maximum (minimum) value:

$$
\begin{gathered}
\mathrm{q}_{\mathrm{m}}=\frac{2}{\mathrm{~N}^{2}}\left(\mathrm{~N}_{\mathrm{A}} \mathrm{N}_{\mathrm{B}}+\mathrm{N}_{\mathrm{A}}^{2}\right) \\
\mathrm{q}_{0}=\frac{2 \mathrm{~N}_{\mathrm{A}} \mathrm{N}_{\mathrm{B}}}{\mathrm{N}^{2}}
\end{gathered}
$$

Note that $\sigma=0$ implies $\eta=0$, however, $\eta=0$ can correspond to $\sigma \neq 0$.

We define the average of some quantity on site $\alpha(\alpha=\mathrm{A}, \mathrm{B})$, the average being taken over all configurational states, as follows:

$$
\left\langle f_{\alpha}\right\rangle=\sum_{\mathrm{j}=0}^{\mathrm{Z}} \mathrm{W}_{\alpha}(j) f_{\alpha}(j)
$$

where $\mathrm{W}_{\alpha}(j)$ is a probability of finding silmultaneously $(Z-j)$ nearest $\alpha$-atoms and $j$ unlike $\alpha$-atoms surrounding a given $\alpha$ site. We have

$$
\mathrm{W}_{\alpha}(j)=\frac{\mathrm{Z} !}{(\mathrm{Z}-\mathrm{j}) ! \mathrm{j} !} p_{\alpha \alpha}^{Z-j} p_{\alpha \underline{\alpha}}^{j}
$$


where $p_{\alpha \alpha}\left(p_{\alpha \underline{\alpha}}\right)$ is the possibility of an $\alpha$ atom (unlike $\underline{\alpha-}$ atom) being nearest neighbour of a given $\alpha$ atom.

Let $\mathrm{V}_{\alpha}(j)$ be the potential energy of an $\alpha$ atom having $(Z-j)$ nearest-neighbour $\alpha$ atoms and $j$ nearest-neighbour unlike $\underline{\alpha}$-atoms. According to Bonnier et al. [6], we assume the following form for the $\alpha$ - atom potential:

$$
\mathrm{V}_{\alpha}(j)=\mathrm{V}_{\alpha}\left(1+a_{1} j+a_{2} j^{2}\right)
$$

where

$$
\mathrm{V}_{\alpha}=\mathrm{V}_{\alpha}(j=0)
$$

Noting that $V_{\mathrm{A}}(j)$ has a minimum at $j=\mathrm{Z}$, one gets from $(6)$ :

$$
V_{\alpha}(j)=\mathrm{V}_{\alpha}+\frac{j(2 Z-j)}{Z^{2}}\left[V_{\alpha}(Z)-V_{\alpha}\right]
$$

We may interpret the formula (7) in a simple way.

When we replace $\mathrm{Z}$ atoms $\mathrm{A}$ surrounding the central atom $\underline{A}$ by $\mathrm{Z}$ atoms $\mathrm{B}$, the energy varies a quantity of $V_{\mathrm{A}}(Z)-V_{\mathrm{A}}$. This energy may be considered as the interaction energy between $\mathrm{Z}$ pairs $\underline{A} A$ and $\mathrm{Z}$ pairs $\underline{A} B$. The interaction energy between a pair $\underline{A} A$ and $\mathrm{Z}$ pairs $\underline{A} B$ is equal to $\frac{V_{\mathrm{A}}(Z)-V_{\mathrm{A}}}{\mathrm{Z}}$. The interaction energy between a pair $\underline{A} A$ and $j$ pairs $\underline{A} B$ is $\left[\frac{\mathrm{V}_{\mathrm{A}}(Z)-V_{\mathrm{A}}}{\mathrm{Z}^{2}}\right] \cdot j$. The interaction energy between $(Z-j)$ pairs $\underline{A} A$ and $j$ pairs $\underline{A} B$ is $\left[\frac{\mathrm{V}_{\mathrm{A}}(Z)-V_{\mathrm{A}}}{\mathrm{Z}^{2}}\right] \cdot j(Z-j)$ and (8) follows.

Substituting (8) into (4) one obtains the average potential of $\alpha$ atom in the form:

$$
\left\langle\mathrm{V}_{\alpha}\right\rangle=\mathrm{V}_{\alpha}+\frac{\left(V_{\alpha}(Z)-V_{\alpha}\right)}{Z}\left(2\left\langle j_{\alpha}\right\rangle-\frac{\left\langle j_{\alpha}^{2}\right\rangle}{Z}\right)
$$

The average values $\left\langle j_{\alpha}\right\rangle$ and $\left\langle j_{\alpha}^{2}\right\rangle$ are calculated according to (4) and may be written in terms of the probability of finding a pair of unlike atoms $\mathrm{p}_{A B}$ as follows

$$
\begin{gathered}
\left\langle j_{\alpha}\right\rangle=\mathrm{Z} \frac{\mathrm{p}_{\mathrm{AB}}}{\mathrm{C}_{\alpha}} \\
\left\langle j_{\alpha}^{2}\right\rangle=\mathrm{Z} \frac{\mathrm{p}_{\mathrm{AB}}}{\mathrm{C}_{\alpha}}+Z(Z-1) \frac{\mathrm{p}_{\mathrm{AB}}^{2}}{\mathrm{C}_{\alpha}}
\end{gathered}
$$

It is convenient to express the probability $\mathrm{p}_{\mathrm{AB}}$ in terms of the long-range order $\eta$ and the correlation factor $\varepsilon_{\alpha \alpha^{\prime}}^{i j}(i, j=a, b)$, which is defined in a standard way [2,3]. We have

$$
\mathrm{p}_{\mathrm{AB}}=\mathrm{C}_{\mathrm{A}} \mathrm{C}_{\mathrm{B}}+\mathrm{b} \eta^{2}+\varepsilon_{A B}
$$

where

$$
\begin{gathered}
\mathrm{b}=\mathrm{C}_{\mathrm{A}} \mathrm{C}_{\mathrm{B}}\left(1-\mathrm{C}_{\mathrm{AA}}-\mathrm{C}_{\mathrm{BB}}\right) \\
\mathrm{C}_{\alpha \alpha}=\frac{\mathrm{Z}_{\alpha \alpha}}{\mathrm{N}} \\
\varepsilon_{A B}=\mathrm{C}_{\mathrm{A}} \mathrm{C}_{\mathrm{AA}} \varepsilon_{A B}^{a a}+\mathrm{C}_{\mathrm{B}} \mathrm{C}_{\mathrm{BB}} \varepsilon_{A B}^{b b}+\mathrm{C}_{\mathrm{A}} \mathrm{C}_{\mathrm{AB}}\left(\varepsilon_{A B}^{a b}+\varepsilon_{B A}^{a b}\right)
\end{gathered}
$$


The configurational energy of the alloy is defined as

$$
\mathrm{E}=-\left[\mathrm{N}_{\mathrm{A}}<V_{\mathrm{A}}>+\mathrm{N}_{\mathrm{B}}<V_{\mathrm{B}}>\right]
$$

and is derived from $(9)-(10)$ to give:

$$
\mathrm{E}=-\frac{\mathrm{NZ}}{2} \cdot\left[\mathrm{C}_{\mathrm{A}} V_{\mathrm{AA}}+\mathrm{C}_{\mathrm{B}} \mathrm{V}_{\mathrm{BB}}+\omega \mathrm{p}_{\mathrm{AB}}+\frac{(Z-1)}{Z}\left(\omega p_{A B}-\frac{\beta}{\mathrm{C}_{\mathrm{A}} \mathrm{C}_{\mathrm{B}}} p_{A B}^{2}\right)\right]
$$

where

$$
\begin{gathered}
\omega=2 \mathrm{~V}_{\mathrm{AB}}-V_{\mathrm{AA}}-V_{\mathrm{BB}} \\
\beta=\mathrm{V}_{\mathrm{AB}}-\mathrm{C}_{\mathrm{A}} \mathrm{V}_{\mathrm{BB}}-\mathrm{C}_{\mathrm{B}} \mathrm{V}_{\mathrm{AA}}
\end{gathered}
$$

The configurational free energy is

$$
F=E-T S
$$

We evaluate the entropy in two cases. First, we consider the system being in the completely disordered phase with zero long-range order parameter. In this case one does not distinguish $a$-site or $b$-site. The calculation is straightforward using the standard method given in textbooks $[3,4]$ :

$$
\begin{aligned}
S= & -\mathrm{Nk}\left\{(1-2 \mathrm{Z})\left(\mathrm{C}_{\mathrm{A}} \ln \mathrm{C}_{\mathrm{A}}+\mathrm{C}_{\mathrm{B}} \ln \mathrm{C}_{\mathrm{B}}\right)\right. \\
& \left.+Z\left[\left(\mathrm{C}_{\mathrm{A}}-\mathrm{p}_{\mathrm{AB}}\right) \ln \left(\mathrm{C}_{\mathrm{A}}-\mathrm{p}_{\mathrm{AB}}\right)+\left(\mathrm{C}_{\mathrm{B}}-\mathrm{p}_{\mathrm{AB}}\right) \ln \left(\mathrm{C}_{\mathrm{B}}-\mathrm{p}_{\mathrm{AB}}\right)+2 \mathrm{p}_{\mathrm{AB}} \ln \mathrm{p}_{\mathrm{AB}}\right]\right\}
\end{aligned}
$$

where $k$ is the Boltzmann constant.

Using (17), (20) and (21) one obtains the configurational free energy for disordered phase as follows:

$$
\begin{aligned}
F= & -\frac{N Z}{2}\left[\mathrm{C}_{\mathrm{A}} \mathrm{V}_{\mathrm{AA}}+\mathrm{C}_{\mathrm{B}} \mathrm{V}_{\mathrm{BB}}+\left(\frac{2 Z-1}{Z}\right) \omega p_{A B}-\left(\frac{Z-1}{Z}\right) \frac{\beta}{\mathrm{C}_{\mathrm{A}} \mathrm{C}_{\mathrm{B}}} p_{A B}^{2}\right] \\
& +N k T\left\{(1-2 Z)\left(\mathrm{C}_{\mathrm{A}} \ln \mathrm{C}_{\mathrm{A}}+\mathrm{C}_{\mathrm{B}} \ln \mathrm{C}_{\mathrm{B}}\right)\right. \\
& \left.+\mathrm{Z}\left[\left(\mathrm{C}_{\mathrm{A}}-p_{A B}\right) \ln \left(\mathrm{C}_{\mathrm{A}}-p_{A B}\right)+\left(\mathrm{C}_{\mathrm{B}}-p_{A B}\right) \ln \left(\mathrm{C}_{\mathrm{B}}-p_{A B}\right)+2 p_{A B} \ln p_{A B}\right]\right\} .
\end{aligned}
$$

We note that when $\eta=0$ the pair probability $p_{A B}$ can be written in terms of short-range order parameter $\sigma$ as:

$$
p_{A B}=\mathrm{C}_{\mathrm{A}} C_{B}(1-\sigma)
$$

The quantities $\mathrm{C}_{\mathrm{A}}$ and $\mathrm{C}_{\mathrm{B}}$ are fixed while $\sigma$ must be adjusted to minimize the free energy. Differentiation of (22) with respect to $\sigma$ gives the following equation for the short-range order parameter:

$$
\ln \frac{\left(\mathrm{C}_{\mathrm{A}}^{2}-\varepsilon_{A B}\right)\left(\mathrm{C}_{\mathrm{B}}^{2}-\varepsilon_{A B}\right)}{\left(\mathrm{C}_{\mathrm{A}} \mathrm{C}_{\mathrm{B}}+\varepsilon_{A B}\right)^{2}}=\frac{\left(\frac{Z-1}{Z}\right)\left(1+\frac{\varepsilon_{A B}}{\mathrm{C}_{\mathrm{A}} \mathrm{C}_{\mathrm{B}}}\right) \beta+\left(\frac{1-2 Z}{2 Z}\right) \omega}{k T}
$$


Now we derive the equilibrium equation for the long-range order parameter, when the system is in the ordered state and the short range correlations can be neglected $\left(\varepsilon_{A B}=\right.$ $0)$. Following the method in [6], it is straightforward to evaluate the entropy. We find:

$$
\begin{aligned}
S= & -\mathrm{Nk}\left\{\nu_{\mathrm{A}}\left(\mathrm{C}_{\mathrm{A}}+\nu_{b} \eta\right) \ln \left(\mathrm{C}_{\mathrm{A}}+\nu_{b} \eta\right)+\nu_{a}\left(\mathrm{C}_{\mathrm{B}}-\nu_{b} \eta\right) \ln \left(\mathrm{C}_{\mathrm{B}}-\nu_{b} \eta\right)\right. \\
& +\nu_{b}\left(\mathrm{C}_{\mathrm{B}}+\nu_{a} \eta\right) \ln \left(\mathrm{C}_{\mathrm{B}}+\nu_{a} \eta\right)+\nu_{b}\left(\mathrm{C}_{\mathrm{A}}-\nu_{a} \eta\right) \ln \left(\mathrm{C}_{\mathrm{A}}-\nu_{a} \eta\right)
\end{aligned}
$$

Using (17), (20) and (25) one gets the configurational free energy for the ordered state:

$$
\begin{aligned}
F= & -\frac{N Z}{2}\left\{\mathrm{C}_{\mathrm{A}} \mathrm{V}_{\mathrm{AA}}+\mathrm{C}_{\mathrm{B}} \mathrm{V}_{\mathrm{BB}}+\left(\frac{Z-1}{Z}\right)(\omega-\beta) \mathrm{C}_{\mathrm{A}} \mathrm{C}_{B}\right. \\
& \left.+b \eta^{2}\left[\omega+\frac{(Z-1)}{Z}(\omega-2 \beta)\right]-\frac{(Z-1)}{Z} \cdot \frac{\beta}{\mathrm{C}_{\mathrm{A}} \mathrm{C}_{\mathrm{B}}} b^{2} \eta^{4}\right\} \\
& +\operatorname{NkT}\left\{\nu_{\mathrm{A}}\left(\mathrm{C}_{\mathrm{A}}+\nu_{b} \eta\right) \ln \left(\mathrm{C}_{\mathrm{A}}+\nu_{b} \eta\right)+\nu_{a}\left(\mathrm{C}_{\mathrm{B}}-\nu_{b} \eta\right) \ln \left(\mathrm{C}_{\mathrm{B}}-\nu_{b} \eta\right)\right. \\
& +\nu_{\mathrm{B}}\left(\mathrm{C}_{\mathrm{B}}+\nu_{a} \eta\right) \ln \left(\mathrm{C}_{\mathrm{B}}+\nu_{a} \eta\right)+\nu_{b}\left(\mathrm{C}_{\mathrm{A}}-\nu_{a} \eta\right) \ln \left(\mathrm{C}_{\mathrm{A}}-\nu_{a} \eta\right)
\end{aligned}
$$

where

$$
b=\nu_{\mathrm{A}} \nu_{b}\left(1-\nu_{a a}-\nu_{b b}\right)
$$

and the equation for the long-range order parameter is given by:

$$
\begin{aligned}
& \ln \frac{\left(\mathrm{C}_{\mathrm{A}}+\nu_{b} \eta\right)\left(\mathrm{C}_{\mathrm{B}}+\nu_{a} \eta\right)}{\left(\mathrm{C}_{\mathrm{A}}-\nu_{a} \eta\right)\left(\mathrm{C}_{\mathrm{B}}-\nu_{b} \eta\right)} \\
= & \frac{\mathrm{Z}\left(1-\nu_{\mathrm{aa}}-\nu_{b b}\right)}{\mathrm{kT}} \cdot\left\{\eta\left[\omega+\left(\frac{Z-1}{Z}\right)(\omega-2 \beta)\right]-\frac{2(Z-1) \nu_{a} \nu_{b}\left(1-\nu_{a a}-\nu_{b b}\right)}{Z \mathrm{C}_{\mathrm{A}} \mathrm{C}_{\mathrm{B}}} \cdot \beta \eta^{3}\right\}
\end{aligned}
$$

The equations (17), (22), (24), (26) and (27) are the main results of the present paper.

The theory presented above is remarkably general and valid for any type of lattice. Once we have calculated the free energy and the order parameter equations, they provide the way to obtain the phase transition critical temperature and the heat capacity given a set of the crystal structure parameters. For the body-centered cubic lattice ( $\beta$ - brass structure) we have:

$$
\begin{gathered}
\mathrm{C}_{\mathrm{A}}=\mathrm{C}_{\mathrm{B}}=\frac{1}{2} ; \mathrm{C}_{\mathrm{AA}}=\mathrm{C}_{\mathrm{BB}}=0 \\
Z=8 ; \mathrm{Z}_{\mathrm{AA}}=\mathrm{Z}_{\mathrm{BB}}^{\overline{B_{B}}} 0
\end{gathered}
$$

while for the face-centered cubic lattice

$$
\begin{gathered}
\mathrm{C}_{\mathrm{A}}=\frac{1}{4}, \mathrm{C}_{\mathrm{B}}=\frac{3}{4} \\
Z=12 ; \mathrm{Z}_{\mathrm{AA}}=0 ; \mathrm{Z}_{\mathrm{AB}}=12 ; \mathrm{Z}_{\mathrm{BB}}=8 ; \mathrm{Z}_{\mathrm{BA}}=4
\end{gathered}
$$

For illustration we consider an $\mathrm{AB}$ alloy (e.g. $\beta$-brass). Using (28) the equation (27) becomes:

$$
\ln \left(\frac{1+\eta}{1-\eta}\right)=\frac{4}{\mathrm{kT}} \eta \omega\left(1-\frac{7}{8} \eta^{2}\right)
$$


The critical temperature is determined by expanding the logarithms in series for small $\eta$ as follows:

$$
2 \eta+\frac{\eta^{3}}{3}+\ldots=\frac{4}{\mathrm{kT}} \eta \omega\left(1-\frac{7}{8} \eta^{2}\right)
$$

Letting $\eta \rightarrow 0$, (31) gives the critical temperature for AB alloy:

$$
k T_{C}=2 \omega
$$

The heat capacity is the derivative of (17) with respect to temperature. Using (28), it gives the jump in discontinuity in heat capacity at $T_{C}$ for $\mathrm{AB}$ alloys as

$$
\Delta \mathrm{C}_{\mathrm{V}}=\frac{12}{29} N k
$$

The temperature dependence of the order parameter may be computed from Eqs. (30) and (32). Analogously, one can obtain the critical temperature and the heat capacity for the face-centered cubic lattice, using equations (17), (27) and (29).

\section{DISCUSSIONS}

It is of interest to compare the above obtained values (32) and (33) with the ones given by the various theories. In the Bragg-Williams approximation $k T_{C}=2 \omega ; \Delta \mathrm{C}_{\mathrm{V}}=$ $\frac{3}{2} N k$ while in the Kirkwood theory $k T_{C}=1,707 \omega ; \Delta \mathrm{C}_{\mathrm{V}}=2,207 N k$; in the quasichemical method $k T_{C}=1,738 \omega[3,4]$. Thus our results give the same value for $T_{C}$ as the Bragg-Williams approximation does, but the jump in discontinuity in heat capacity is smaller than the other theories. In the Bragg-Williams approximation the configurational energy is given by [1-3]:

$$
E_{0}=-\frac{N Z}{2}\left(C_{A} V_{A A}+C_{B} V_{B B}+p_{A B} \omega\right)
$$

Comparing (34) with (17) one can see that the surrounded atom theory gives the correction $\frac{(Z-1)}{Z}\left(p_{A B} \omega-\frac{\beta}{\mathrm{C}_{\mathrm{A}} \mathrm{C}_{\mathrm{B}}} p_{A B}^{2}\right)$ for every bond to the Bragg-Williams energy due to the interactions between the bonds. Comparing with experiment our results as well as the Bragg-Williams approximation reproduce the general feature of the order-disorder transition. However, the experimental data show that the order-disorder contribution to the heat capacity does not instantly vanish immediately above $T_{C}$ [2-3]. The BraggWilliams theory does not show a residual short-range order heat capacity above the critical temperature, while the surrounded atom theory takes into account the short-range order contribution (Eqs. (17) and (20)). The surrounded atom method clearly is an improvement over the Bragg-Williams one, but not sufficiently so to yield the good agreement with experiment.

There are several possible ways to improve the current work. The most obvious one is to go beyond the nearest-neighbour approximation for the interaction energies. The second one is to take into account the vibrational contribution to the free energy. The third one concerns the calculation of the bond number. In the Bragg-Williams approximation the site occupation probabilities are taken to be independent of each other. In our work, 
as in the quasi-chemical approximation, this is improved somewhat by counting bonds, but these bonds then considered to be independent.

\section{ACKNOWLEDGEMENTS}

The authors are grateful to Professor Nguyen Huu Minh (Hanoi National University of Education) for the stimulating suggestions.

\section{REFERENCES}

[1] W. L. Bragg and E. J. Williams, Proc. Roy. Soc. A145 (1934) 699.

[2] T. Muto and Y. Takagi, Solid State Phys., F. Seitz and D. Turnbull (Eds.), 1 (1955) 193.

[3] L. A. Girifalco, Statistical Mechanics of Solids, Oxford University Press, 2002.

[4] F. Ducastell, Order and Phase Stability in Alloys, Elsevier Science, New York, 1991.

[5] E. Bruno et al, Phys. Rev. B77 (2008) 155108 and references therein.

[6] E. Bonnier et al, J. Chem. Phys., 64 (1967), 261; Adv. Phys. 63 (1967) 523.

[7] Nguyen Huu Minh, J. Phys. 2 (1977) 8-14; 4 (1977) 24-27 (in Vietnamese).

Received 25 April 2011. 